\title{
TWO CASES OF ASPERGILLUS INFECTION OF THE CENTRAL NERVOUS SYSTEM
}

\author{
BY \\ SUBRAMANI IYER, PHIEIP R. DODGE, and RAYMOND D. ADAMS \\ From the Neurological Unit, Boston City Hospital, the Neurological Service, Massachusetts General Hospital, \\ and the Department of Neurology, Harvard Medical School
}

Fungus infections of the nervous system are admittedly rare and the report of additional cases can be justified only if they demonstrate new facts. We believe this to be true of the following two cases. They present certain clinical features not described previously in association with aspergillosis and also exhibit an unusual combination of pathological changes which aid in the interpretation of the pathology of other types of chronic inflammatory disease of the central nervous system.

\section{Case Reports}

Case 1. - The patient, a 23-year-old man, was admitted to the Tokyo Army Hospital on February 18, 1951, because of increasing headache, double vision, and stiffness of the neck. The symptoms had begun in October, 1949, and the first complaint was severe aching pain over the maxillary and frontal sinuses. These pains persisted intermittently until June, 1950, and were followed by a constant burning pain in the right occipital region. The latter was most severe in the morning and in the late evening. There was no accompanying nausea or vomiting and change of posture did not influence the pain. The pain ceased about October, 1950.

In November, 1950, while in Korea, the patient awoke one morning with stiffness and aching in the left shoulder and left side of the neck. The ache was relieved by inclining the head to the left. Weakness of the left shoulder and upper arm was also noticed about this time and these symptoms progressed until January, 1951. The aching pain then ceased but weakness and also a stiffness of the left shoulder and upper arm remained. Diplopia was experienced for the first time on February 4, 1951. From the beginning it was worse on lateral gaze, and it gradually increased in severity. At times there was an associated dizziness but never a true vertigo. Unsteadiness of gait was also noted about this time and became progressively worse. On February 10,1951 , the patient began to suffer with severe occipital and bitemporal headaches, and boring pains behind the eyes. After the headache had continued for eight days he was admitted to the hospital. Twenty pounds in weight had been lost during the course of the illness.

A review of the past history contributed relatively little of value. The patient was born and had lived most of his life in Mississippi. There had been no serious past illnesses. In the year and a half before admission he had been stationed in China, Guam, Okinawa, Japan, and Korea. The family history was not contributory.

On admission the patient came under the care of one of us (P.R.D.). It was observed that he was rather thin but he did not appear to be particularly ill. Temperature, pulse, and respirations were normal and blood pressure was $120 / 80$. The skin was covered with numerous pigmented moles and a large café-au-lait spot was noted in the right axilla. The superficial lymph nodes were not palpable. There were no abnormalities of the ears, nose, throat, and bony sinuses, and the heart, lungs, and abdomen were not remarkable.

The patient was alert, rational, and cooperative. There was papilloedema, estimated at two to three diopters. There were retinal haemorrhages bilaterally. The visual fields were of normal configuration except for an enlarged blind spot on the left ; visual acuity was within normal limits. The pupils were round, regular and equal in size, and reacted normally. There was bilateral lateral rectus weakness which was more marked on the left. No nystagmus was elicited. All other cranial nerves functioned normally. All of the muscles of the left shoulder girdle and the flexors of the left arm were weak and atrophic and numerous fascicular twitchings were seen in them. The left biceps reflex was definitely diminished and the left supinator reflex questionably so ; the triceps reflexes were equal and active. The left knee jerk was slightly more active than the right and the ankle jerks were equal. The plantar reflexes were flexor. There was no defect in sensation. Movements of the limbs were well coordinated; the gait was normal and Romberg's sign was absent.

Laboratory Data.-The white blood cells numbered 8,300 with a normal differential count. The sedimentation rate was $5 \mathrm{~mm}$. at the end of one hour, and the haematocrit value was $52 \%$. Serological tests for syphilis were negative. Urine analysis was within normal limits. Radiographs of the chest did not reveal any remarkable findings. A lumbar puncture was not performed on admission because of the severe papilloedema and headache. 

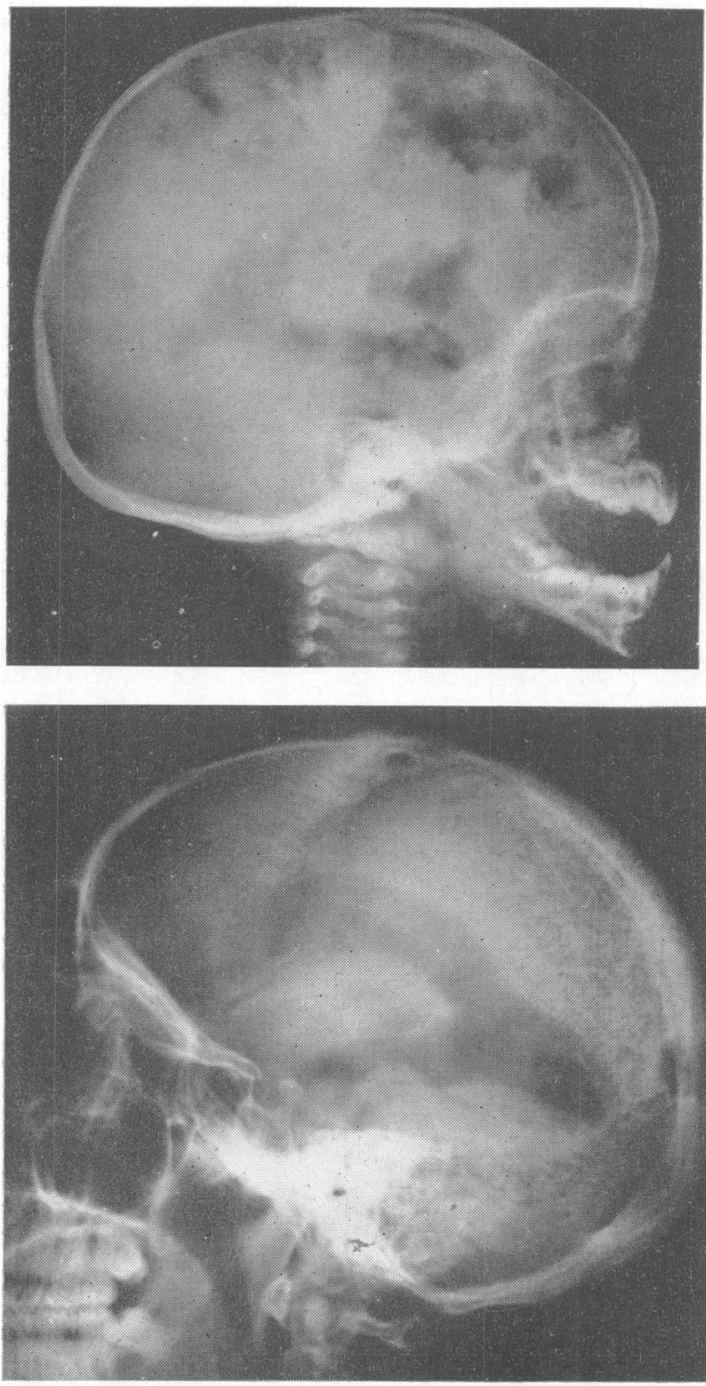

Course.-The clinical findings remained the same except for the appearance of a mild antero-posterior rhythmical titubation of the head. A ventriculogram was performed on February 27, 1951, and a symmetrical enlargement of the lateral and the third ventricles and the upper portion of the aqueduct of Sylvius was demonstrated (Fig. 1B). The ventricular fluid was blood-tinged (traumatic) and contained $41 \mathrm{mg}$. of protein per $100 \mathrm{ml}$. The same day a suboccipital craniectomy was performed by Lt.-Col. A. M. Meirowsky. The dura was tense and the cisterna magna contained a small amount of normalappearing fluid. The arachnoidal membrane over the dorsal surface of the lower medulla and upper cervical spinal cord was thin and transparent. A good view of the fourth ventricle was obtained and a catheter could be passed easily from the fourth ventricle through the
FIG. 1.-Pneumoencephalograms.

A. Case 2. There is incomplete filling of ventricles and moderate amounts of air over the surface of the brain. Wisps of calcium can be seen behind the lateral ventricle.

B. Case 1. The lateral ventricles are moderately dilated.

FIG. 2.-Necropsy material from Case 2 .

A. Coronal section through the parietal lobes. Note the extensive destruction of brain tissue and the numerous cavities filled with a gelatinous material.

B. The same distended cavities in the fronto-parietal region as seen from the surface of the hemisphere.
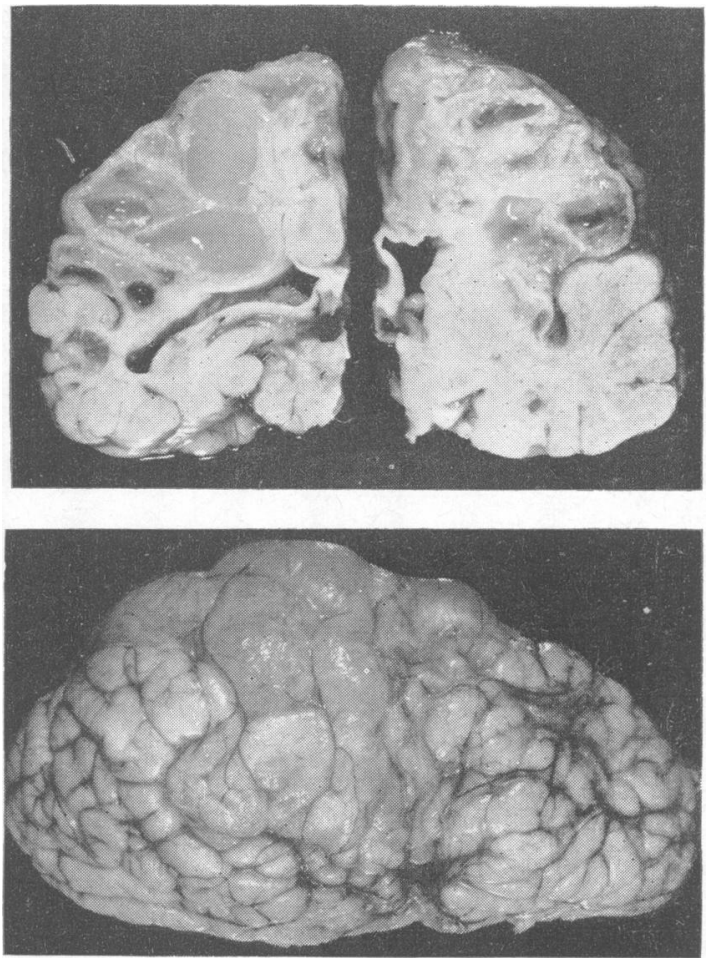

aqueduct. A smooth, lobulated, pearly-yellow mass was seen lying ventro-lateral to the lower medulla and upper cervical spinal cord. Two large vessels were seen to enter the mass. The mass was firm and adherent to the medulla ; it was thought to be a benign neoplasm. It was considered to be inoperable because of its ventral location. Biopsies taken at this time and at a subsequent operation two days later disclosed a chronic granulomatous inflammation with marked fibrosis, round cell infiltration, and foreign-body giant cells. In the second biopsy the hyphae of a fungus were seen in a necrotic focus. The microscopic appearance of these hyphae suggested ustilagomycosis (Moore, Russell, and Sachs, 1946) but cultures taken at the time of the operation remained sterile.

Following operation the patient showed no significant 
FIG. 3.-Photomicrographs of sections from Case 1 stained with haematoxylin and eosin.

A. Coronal section through the medulla at the level of the inferior olivary nuclei showing the mass of fibrous and granulomatous tissue lying ventral to the pyramids.

B. The meningeal mass at the level of the cervical cord. There is a small granuloma containing inflammatory cells (some degenerating), histiocytes, and multinucleated giant cells. Note the dense fibrous capsule.

c. Same level as B but showing a rather sparse infiltration of inflammatory cells and an abundance of dense collagenous connective tissue.
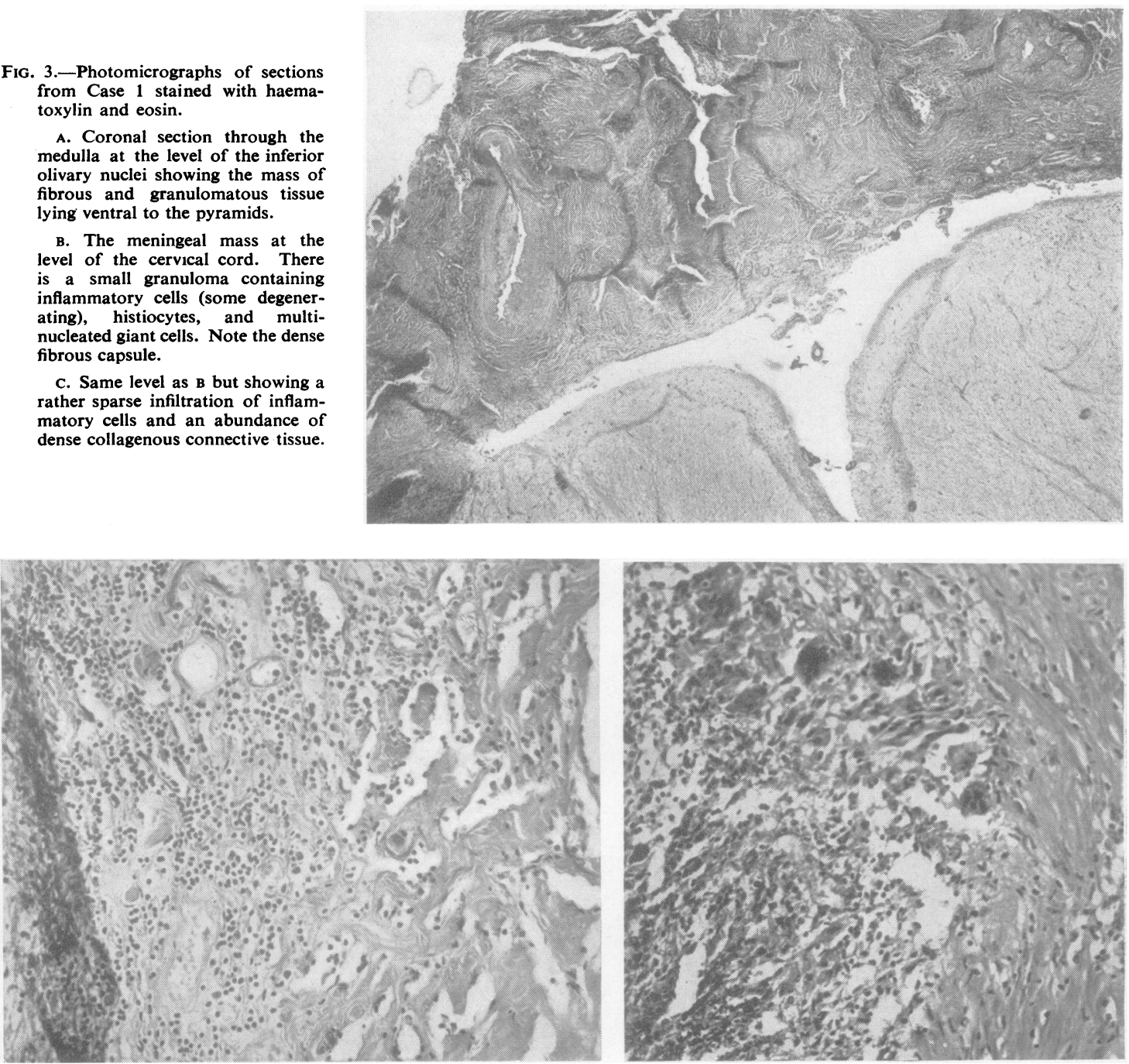

alteration in his vital signs. The diplopia persisted and ocular movements were now greatly impaired; he was, in fact, unable to move either eye horizontally and on attempting extreme left gaze only a few slight unsustained adduction movements of the right eye beyond the midline were noted. Vertical movements were unaffected. The pupils were equal and reacted normally. There was gradual regression of the papilloedema. Three days after the operation the spinal fluid contained 40,000 erythrocytes and 351 leucocytes per $100 \mathrm{c}$. $\mathrm{mm}$.; the protein was $110 \mathrm{mg}$. per $100 \mathrm{ml}$. Cultures were again negative.

On the evening of March 16, 1951, severe generalized headache and pain in the neck returned for the first time since operation. The patient became lethargic and slow to respond. The left knee and ankle jerks were hyperactive, the abdominal reflexes were absent on the left, and the left plantar reflex was extensor. Shortly thereafter he developed retention of urine which required constant drainage of the bladder.

On March 17, 1951, a ventricular puncture was performed, and the intraventricular pressure was found to be normal. The ventricular fluid contained $55 \mathrm{mg}$. of protein and $55 \mathrm{mg}$. of sugar per $100 \mathrm{ml}$. The lumbar puncture was repeated and an almost complete block in cerebrospinal fluid dynamics was found. The lethargy. continued for several days and an intermittent fever up to. $101^{\circ} \mathrm{F}$. was recorded. When examined on March 25, 1951 , the patient was disorientated. The papilloedema had subsided. A left Horner's syndrome was noted for the first time. The other findings were unchanged, The patient died at Tripler Army Hospital, Hawaii, while on his way to the United States. 
Post-mortem Examination.-The necropsy was performed approximately 30 hours after death.* A moderate degree of emaciation was evident at this time. The cranial operative incisions were well healed. A small decubitus ulcer was present over the posterior aspect of the right iliac crest. There were no significant gross anatomical findings outside the central nervous system ; in particular, the lungs, heart, liver, spleen, and the gastro-intestinal tract were not remarkable either by gross or microscopic examination.

The dura was adherent to the galea aponeurotica in the region of the operative defect in each parietal bone and in the occipital region. At the base of the brain there were a few adhesions between the dura and arachnoid. The tentorium was also adherent to the upper surface of the cerebellum. The cerebral hemispheres were symmetrical and of normal appearance from the external aspect. The leptomeninges and vessels on the supero-lateral surfaces of the brain were normal. At the base of the brain on the left side the trigeminal, facial, and auditory nerves were enveloped by a mass that was situated anterior to the brain-stem. It had produced a pressure defect to a depth of $0.7 \mathrm{~cm}$. on the surface of the brain-stem over an area that measured $2.5 \times 1.5 \mathrm{~cm}$. A series of sections through the cerebral hemispheres revealed moderate dilatation of the lateral and third ventricles and the aqueduct of Sylvius. The basal ganglia and the choroid plexuses appeared normal. The meningeal mass was firm and fibrotic. When the brain-stem was sectioned the asymmetry of the basilar pons, the upper medulla, and the left quadrangular lobe of the cerebellum was more clearly seen. Below the level of the medulla the mass thinned out to such a degree that it was no longer visible. The spinal leptomeninges were slightly opaque and thickened but no lesions were noted in the substance of the cord, and the spinal roots were of average size.

Sections of the spinal cord, medulla, pons, midbrain, cerebellum, and the cerebral cortex were prepared for microscopic examination by staining with haematoxylin and eosin.

Over the ventral surface of the upper cervical spinal cord the thickened leptomeninges formed a dense mass composed of several layers of collagenous connective

* Necropsy was performed by Lt. Donald G. McLeod who very kindly made available to us the gross findings and microscopic sections.
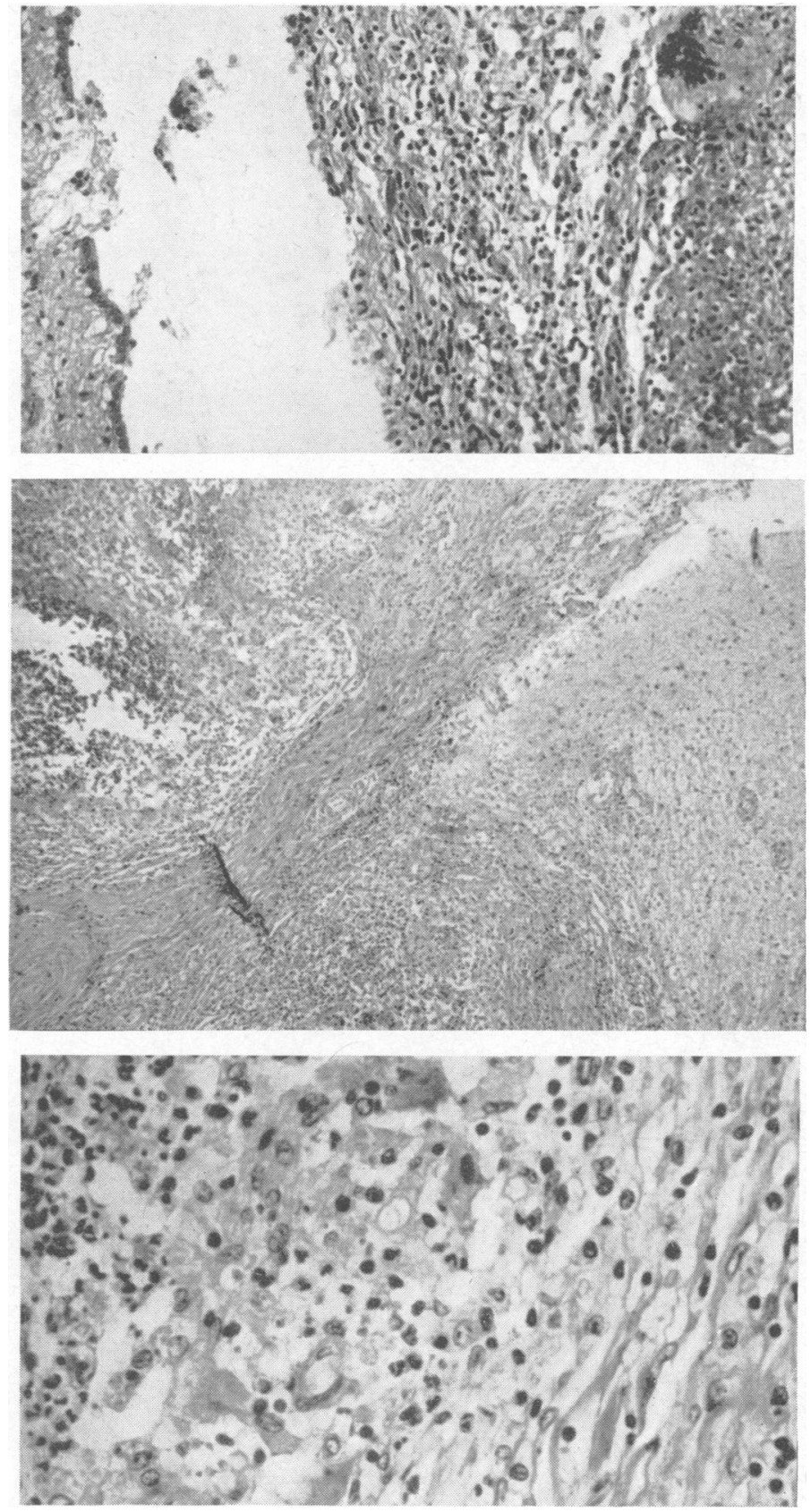

FIG. 4.-Photomicrograph of section from Case 1 stained with haematoxylin and eosin.

A. The ependymal lining of the floor of the fourth ventricle is shown on the left. The tissue on the right is a mass of astrocytes, histiocytes, and fibroblasts and partially fills the fourth ventricle and is attached to its floor in several places. One giant cell is seen in the right upper corner.

B. Section at the junction of pons and midbrain to show a granulomatous mass invading the subpial tissues.

C. Same level as B. The fragmented nuclei on the left represent a small abscess. Fibroblasts, lymphocytes, plasma cells, and histiocytes are seen on the right and in the centre. 

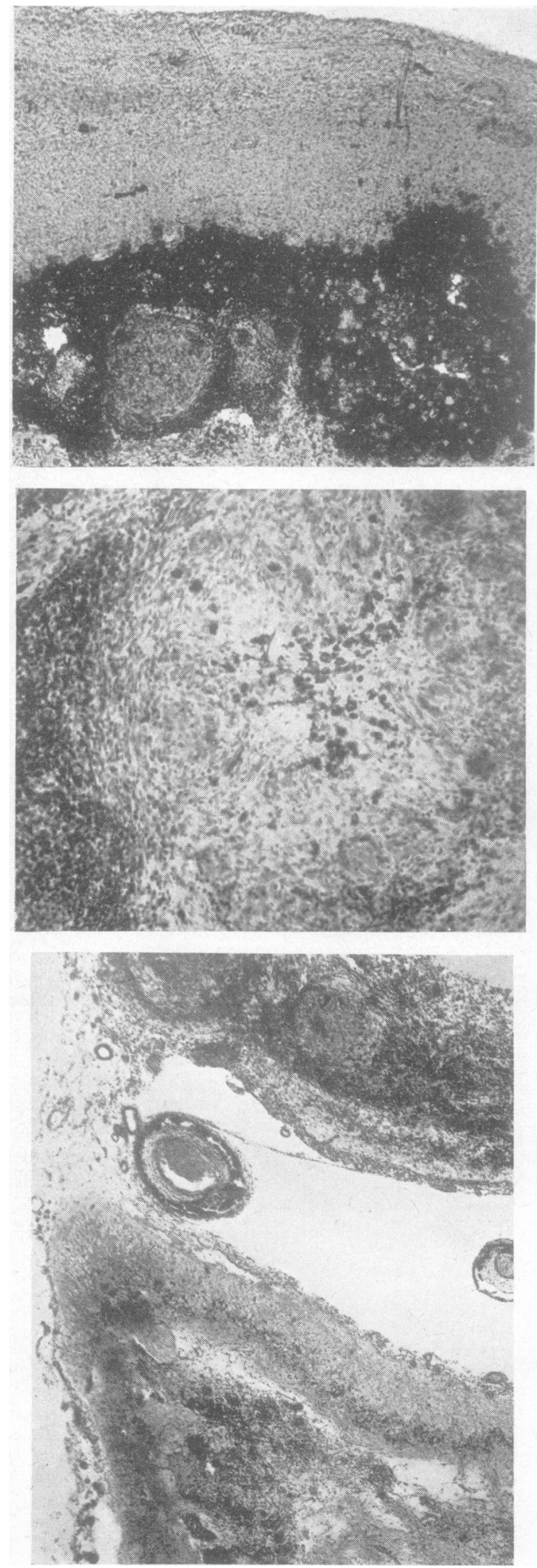

tissue. Small abscesses and granulomas were embedded within this membrane. In the centre of the abscesses there was necrosis and only the debris of leucocytes and other cells remained. These abscesses were encapsulated by fibroblasts; also occasional giant cells and numerous inflammatory cells were seen (Fig. 3B). Several granulomas were observed, in some of which there were foreign-body giant cells and epithelioid cells surrounded by a peripheral zone of lymphocytes and plasma cells. Fungi were not seen either in the abscesses or granulomas at this level. The nerve roots in the thickened leptomeninges were not damaged. Elsewhere in this membrane small blood vessels were dilated and diffuse and focal infiltration of inflammatory cells were observed (Fig. 3c). There were no changes in the substance of the spinal cord other than a few mononuclear cells around the central canal.

Sections through the junction of the pons and medulla showed a similar dense granulomatous mass ventral to the pyramids (Fig. 3A). In addition to numerous small abscesses and granulomas several thrombosed and recanalized arteries were seen at this level. Branched, septate, highly refractile rods were seen in the centres of the abscesses (Fig. 6A). There were focal collections of microglia under the pia in the ventral portion of the medulla. The floor of the fourth ventricle was covered by a granulomatous mass which replaced the ependymal lining in some places and buried islands of ependymal cells in others (Fig. 4A). At one point the inflammatory process extended through the ependymal lining into the tegmentum of the medulla. There was hyperplasia of the endothelial and adventitial cells of numerous small vessels in the sub-ependymal zone, and the VirchowRobin spaces of some of these vessels contained mononuclear cells. A diffuse microglial proliferation was seen in the dorsal tegmentum of the pons. Occasional hyphae were found in the granulomatous formations in the ependyma but there were none in the sub-ependymal layer. The ependymitis appeared to be fairly recent, especially in one side of the fourth ventricle where a mass of fibrin and leucocytes was found attached to the ependyma.

Sections through higher levels showed the fibrogranulomatous mass to have invaded the brain stem in two places. One such lesion, situated in the basis pontis at the junction of the pons and midbrain, is pictured in Fig. 4B. Here within the pons there was a small abscess walled off from the rest of the pons by a zone of collagenous connective tissue containing numerous lymphocytes, occasional plasma cells, polymorphonuclear

FIG. 5.-Photomicrographs of sections from Case 2 stained with cresyl violet.

A. Cerebral cortex : the black material is calcium and several small granulomas are visible. The nerve cells in the cortex are replaced by astrocytic glia.

B. Same level as A, demonstrating densely cellular granulomas with one large giant cell. Collections of fungi with Hülle cells can be seen in the centre of the granuloma on the right.

c. Note the extreme destruction of the cerebral cortex and subcortical white matter and the calcification, gliosis, and granulomas. 


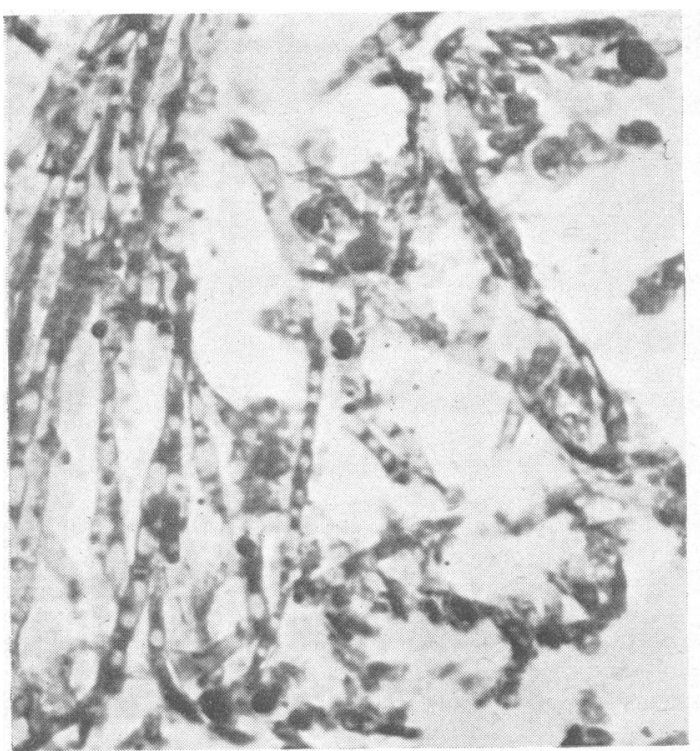

$6 \mathrm{~A}$

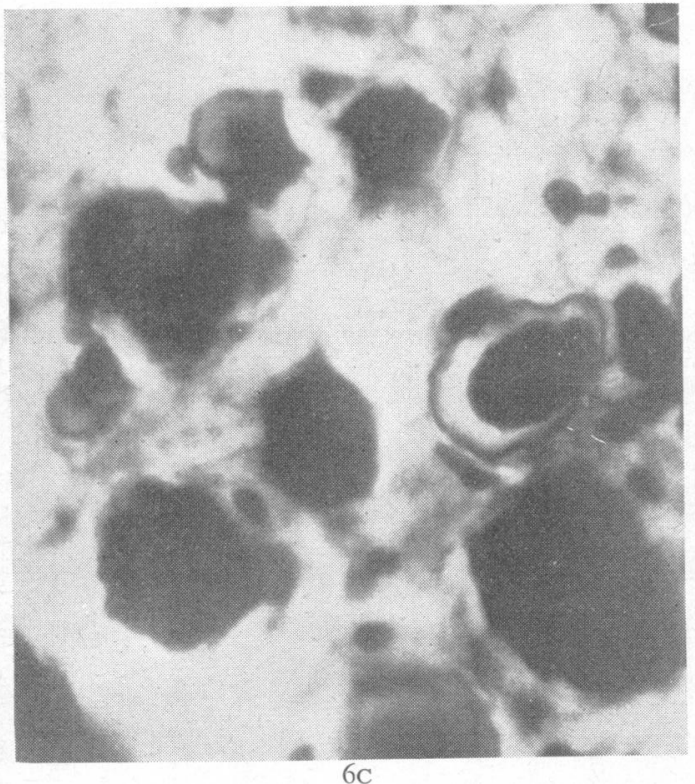

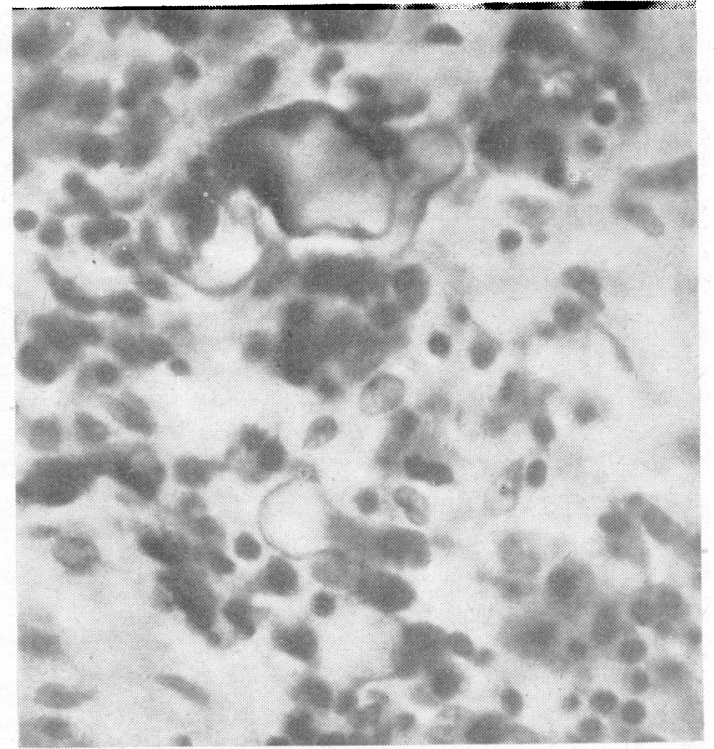

6B

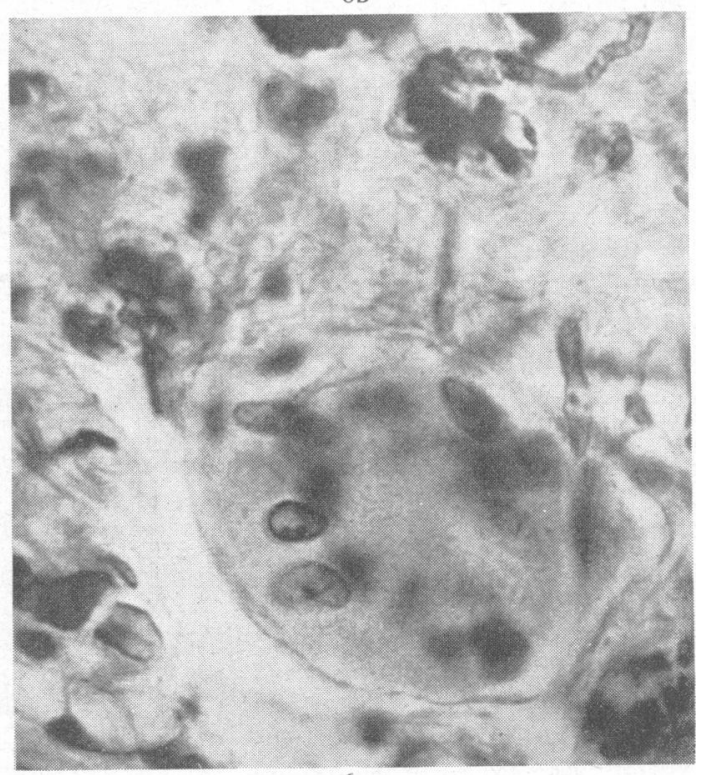

6D

FIG. 6.-Photomicrographs of sections from Cases 1 and 2 showing fungi.

A. Case 1. The refractile, septate, branching hyphae of aspergillus from the granulomatous mass.

B., C., and D, Case 2. Note the fungi of various shapes and sizes. The black masses represent the Hülle cells of aspergillus.

leucocytes, and small blood vessels (Fig. 4c). An increased cellularity and vascularity of the tectum, and an ependymitis of the aqueduct of the Sylvius which contained cellular detritus and an enormous number of fungi, were other striking findings at this level.

Sections of the cerebellum revealed only a mild leptomeningitis with lymphocytes, mononuclears, and a few microglial phagocytes. Loss of Purkinje cells and proliferation of the Bergmann glial cells were noted in a few folia and in one place there was an abscess with centrally placed fungi extending from the meninges into the molecular layer. Similarly, sections through the cerebral hemispheres revealed only a slight thickening of the leptomeninges with moderate infiltration by 
mononuclear cells. In the sub-ependymal zone of the lateral ventricle there were small dilated and "cuffed" vessels.

An abundant growth of Aspergillus fumigatus was obtained from cultures of the subarachnoid exudate.

Before operation the clinical symptoms were thought to indicate a space-occupying extramedullary lesion of the posterior fossa and upper cervical canal on the left side. Tumour and chronic adhesive arachnoiditis were considered likely aetiological possibilities.

Isolation of the aspergillus was not accomplished while the patient was living, although repeated cultures for fungus were performed on cerebrospinal fluid from the lumbar sac, ventricle, and cisterna magna. Recovery of Aspergillus fumigatus at necropsy, however, did not prove that it was the causative agent, for, in view of the known ubiquity of the fungus, the possibility of a post-mortem contaminant or a secondary invader could not be dismissed. We believe the presence of fungi with typical morphology in the centre of abscesses and granulomas, found in both the biopsy specimen and the necropsy material, left little doubt that we were dealing with an example of aspergillus infection of the central nervous system.

In correlating the clinical with the pathological findings it is probable that the burning pain in the right occipital area reflected early involvement of the upper cervical nerve roots. As the disease progressed more roots were implicated, accounting for the pain in the neck and left shoulder, muscular weakness, atrophy, and diminished left biceps reflex. At what stage in the disease the obstruction to the flow of cerebrospinal fluid occurred could not be determined. Presumably, it happened some time before the onset of headache, papilloedema, and diplopia. Since the aqueduct of Sylvius was patent it must be assumed that the hydrocephalus resulted from fibrous thickening of the basilar leptomeninges with obstruction of the foramens of Magendie and Luschka. The lesions responsible for the hydrocephalus were not demonstrated microscopically because adequate sections through the proper levels were not available.

Invasion of the brain-stem occurred in at least two places, yet it was so slight that only minimal symptoms and signs of brain-stem involvement were produced. Pressure effects must also have been minimal since the extrinsic mass, even at the time of death, was not large. It is surprising that there was not more clinical evidence of involvement of the cranial nerves that were implicated in the inflammatory mass.

Outside the central nervous system no sign of aspergillus infection was demonstrated. The symptoms of sinusitis at the onset of the illness suggested one possible route of invasion by the fungus. Another, and perhaps more likely pathway by which the central nervous system may have been infected, is by direct extension from the ear to the base of the brain where the pathological process was localized. Aspergillus is stated to be a common cause of external ear infection (Whalen, 1938). However, since neither the sinuses nor the ears were cultured for fungi at necropsy, the route of entry remains unproved.

Case 2.-A coloured boy, aged 10 months, was brought to the Boston Floating Hospital because of intermittent spasms of the trunk and limbs, rigidity of the hands and arms, and inability to support the head.

The symptoms had begun at the age of 4 months when the patient became unusually irritable. He cried frequently and seemed generally dissatisfied. At about the same time there was the onset of peculiar spells consisting of a sudden shriek with upward turning of the eyes and stiffening and jerking of all four extremities. These occurred as often as six to seven times a day and each attack lasted two to three minutes. The parents were not sure whether or not consciousness was lost during these spells. Thereafter a noticeable change in the patient's behaviour was noted. Whereas he had been lively, had manifested pleasure by cooing and squealing, had held up his head fairly well, had attempted to pull himself to a sitting position, and had been able to manipulate toys, he was now content to lie passively in his crib taking less and less notice of what went on around him. He continued to eat well and cut two teeth. The lower extremities were noted to have become quite stiff; the upper ones less so. In the weeks preceding admission the patient had become quite apathetic.

Birth had been normal at full term and the weight at birth was $5 \frac{1}{2} \mathrm{lb}$. His body was well proportioned. He breathed without any delay and responded well during the first days of life. During the first four months the patient's appetite was good; he gained weight and developed as rapidly as two older siblings aged $4 \frac{1}{2}$ and 2 years who were regarded as normal. The diet was probably adequate. The family history contributed nothing. No jaundice or haemorrhagic tendency had been observed. In addition to two older siblings there was a third, 2 weeks old, who was also normal.

When first examined after admission to the hospital the patient was noted to be well nourished and about average size for this age. He lay continuously on the side with the neck slightly extended, the arms flexed, and the legs extended. The temperature was $98-99^{\circ} \mathrm{F}$., the pulse ranged between 100 to 120 , and respiration was 30 per minute. When left alone he lay dormant; if molested in any way he whimpered. The head was of normal size and shape. The anterior fontanelle was flat and measured $2.5 \mathrm{~cm}$. in width. Auditory and visual stimuli seldom evoked any response but touching 
or pinching the skin of the face and neck brought about poorly directed squirming movements of all parts of the body. A dangling toy failed to attract his attention but on one occasion his eyes were observed to follow a flashlight and moved conjugately from side to side. The pupils were round and equal and reacted to light promptly. The optic fundi and external ears were normal. One examiner noted that he seemed to hearken when his name was called. He sucked vigorously and retained his food. When pulled to a sitting position his head lolled helplessly indicating, amongst other things, that there was no stiffness of the neck. The skeletal muscles were firm and strong. The arms and legs were spastic, with hyperactive deep tendon reflexes and bilateral extensor plantar responses. Startle caused movements of all four extremities. The limbs were withdrawn from painful stimuli. Tonic neck reflexes were not elicited. There was no abnormality of the spine. The general physical examination was within normal limits except for enlarged tonsils and a grade I systolic cardiac murmur at the third left interspace.

Laboratory Data.-The leucocytic count was 8,900 per c. $\mathrm{mm}$. (34\% neutrophils, $48 \%$ lymphocytes, $7 \%$ eosinophils, $9 \%$ monocytes, and $1 \%$ blast forms). The red cell count was 5.5 million per c. mm. and the haemoglobin $14 \mathrm{mg} . \%$. Urine analysis disclosed no abnormal findings. The cerebrospinal fluid contained $60 \mathrm{mg}$. of protein and $69 \mathrm{mg}$. of sugar per $100 \mathrm{ml}$. No cells were seen and the Hinton test was negative on the blood and cerebrospinal fluid. On culture of the cerebrospinal fluid there was no growth of bacteria or fungi after four days. Fluid from the subdural space contained $38 \mathrm{mg}$. of protein per $100 \mathrm{ml}$. and there were no cells. Radiographs of the chest and hips were not remarkable, but those of the head revealed several radio-opaque densities within the substance of both cerebral hemispheres (Fig. 1A). A toxoplasmin skin test was performed on the infant and his mother. The infant did not show any reaction, but the mother had a questionably positive one.

Course-The patient was placed under observation for 22 days during most of which time he remained in the same stuporous, fretful state. A survey by a psychometrist revealed that only 4 and 6 weeks' tests could be accomplished but testing was so difficult that the results were not thought to be reliable. A pneumoencephalogram was done but had to be discontinued because of a generalized convulsion. The lateral ventricles were dilated and air had accumulated in the subdural and subarachnoid space in amounts suggestive of atrophy of the brain (Fig. 1A). An electroencephalogram showed slow asynchronous waves of high voltage in all leads without a trace of alpha rhythm and was interpreted as abnormal.

The patient's condition changed relatively little during the hospital stay and he was discharged unimproved. In the following two weeks he failed rapidly, became pale, stuporous to semicomatose, and unable to take nourishment. On the morning of December 8, 1948, he was admitted to the Boston City Hospital but died 15 minutes after admission, before any further examination could be carried out. He was 11 months of age at death.

Post-mortem Examination.-A necropsy was performed approximately 26 hours after death. At this time general nutrition and development of the body were judged to be within normal limits. There were no abnormal findings outside the central nervous system. Microscopic examination of the lungs, liver, spleen, heart, kidneys, gastro-intestinal tract, and bone marrow revealed normal findings.

The brain was of average size but grossly abnormal. There were numerous fibrinous adhesions between the cranial dura and the arachnoid membrane. In many places, particularly the central portions of the right cerebral hemisphere, the right temporal lobe, and the left frontal and occipital lobes, the brain tissue was destroyed and replaced by numerous cavities. These cavities ranged from a few millimetres to $2.5 \mathrm{~cm}$. in size, were separated by strands of connective tissue, and contained a grey, gelatinous fluid that clotted on formalin fixation (Figs. 2A and 2B). The cerebral cortex in many of the affected areas was reduced to a thin, grey membrane, and the adjacent arachnoid was thickened and slightly opaque. The tissue between the cavities was grey-brown.

On frontal section the lesions were observed in both grey and white matter. The claustrum and putamen were involved in at least one place. The lateral ventricles were dilated but their walls appeared to be intact. Sections of the brain-stem and cerebellum revealed that the pyramids were smaller than normal and greyish. No lesions were seen in this part of the brain.

Upon microscopic examination it was found that in several sections of the cerebral cortex there was a thickening of the leptomeninges and a moderate amount of cellular exudate in the subarachnoid space. Most of the cells were either lymphocytes or large monocytes, but occasional plasma cells and multinucleated macrophages were seen. The exudate was denser in the depths of the sulci than over the surface of the cortex. There was a thick layer of glial cells in the cerebral cortex just beneath the pia. In the depths of the cortex numerous granulomatous foci, either single or conglomerated into large granulomatous zones (Figs. 5B and 5C), were seen. The centre of the small granulomas was formed of a single or rarely more than one foreign-body giant cell, containing as many as 20 to $\mathbf{3 0}$ nuclei, and epithelioid cells. Fibroblasts surrounded them and in the periphery there were numerous lymphocytes, a few plasma cells, microglial histiocytes, and monocytes. The larger granulomas contained a mixture of fibroblasts and numerous giant cells and inflammatory cells. An interesting finding was the presence of a large number of blue-staining rods which under high magnification appeared tubular. They were indistinctly septate and in places were branched; many of them had bulbous ends. They were gathered in tangled masses or were present singly, and in certain sections a collection of rounded blue bodies, resembling the bulbous terminations of these rods, was seen. These structures were present in the centres of the 
granulomas and were free in the adjacent cortex (Figs. 6B, 6c, and 6D). Also, large hyaline oval or round bodies with translucent borders were encountered. These bodies (Hülle cells) were seen both within and adjacent to the granulomas (Fig. 6c). The blood vessels around the lesions did not appear to be affected, but calcification of the media and subintimal layer was seen in some and easily confused with the aforementioned rods. Calcified particles (Fig. 5A) were found near many of the granulomatous masses. This calcification was most dense in areas where the lesions were fibrotic. The numerous cavities contained an amorphous pinkstaining material. In regions where the lesions were present all of the neurons had disappeared, leaving behind only a framework of proliferated astrocytes. The underlying white matter showed cavitation, loss of myelin, and a fibrous gliosis.

Frontal sections through the basal ganglia disclosed similar lesions in the claustrum, the external capsule, putamen, internal capsule, and, to a slight extent, in the thalamus, subthalamus, and midbrain. The ventricles were not involved, and the ventricular ependyma and choroid plexuses were normal. There were no lesions in the lower part of the brain-stem, cerebellum, or spinal cord.

Sections through the dura mater revealed a partially organized haemorrhage on its inner surface.

The fresh brain material was inoculated in several iaboratory animals none of which ever developed lesions of toxoplasmosis. Routine cultures for bacteria were also negative. No attempt was made to culture a fungus because this possibility was not considered at the time of necropsy.

Clinically this patient was apparently normal till about the age of 4 months when the behavioural regression and epilepsy began. Throughout the entire course of the illness there were signs of pyramidal tract disease bilaterally, in the form of weakness and spasticity of the legs and to a lesser extent of the arms. These facts when considered with the cerebral calcifications, abnormal electroencephalogram, and elevated cerebrospinal fluid proteins suggested clinically that the disease process involved both cerebral hemispheres, and was probably in the nature of a meningo-encephalitis. Toxoplasmosis was considered the most satisfactory aetiological explanation, despite the lack of family history and negative skin tests.

At necropsy the findings were those of a chronic granulomatous meningo-encephalitis. Microscopically the lesions differed from those of toxoplasmosis described by several authors (Wolf and Cowen, 1937; Wolf, Cowen, and Paige, 1939). The presence of well-formed granulomatous giant cells and the absence of any changes in the ependyma and choroid plexuses were clearly inconsistent with a toxoplasmic encephalitis. The examination of numerous sections of the brain and other organs failed to reveal toxoplasma. The retinae were not available for microscopic study but had been normal upon opthalmoscopic examination. Furthermore, inoculation of the brain tissue of the patient into laboratory animals failed to produce toxoplasmosis.

Tuberculosis and syphilis were excluded as possible causes of the pathological lesions, because caseation necrosis did not occur in any of the lesions, particularly the large ones, and there was no arteritis in the vessels about the lesions. Also against syphilis was the absence of involvement of extraneural tissues and the negative Hinton and Wassermann tests.

The tangles of blue-staining rods in and around the lesions were identified as fungi belonging to the group of Hyphomycetes, and the presence of the hyaline cells, which were later identified as Hülle cells (Thom and Raper, 1945), strongly suggested their being aspergilli. A mycologist, Dr. George Foley, kindly examined the sections and reported to us that the morphology of the septate hyphae with conidiophore structures and mycelia is characteristic af aspergillus. He stated that the large densely stained rounded bodies surrounded by a capsule-like halo were probably Hülle cells of aspergillus. The exact origin and function of these cells is unknown.

The chronicity of the lesions in this case indicated that they may have been present before the onset of symptoms. It is interesting to speculate whether this could be an instance of intra-uterine infection. Cases of aspergillus infection of the genital tract have been recorded (Goldstine, 1933 ; Weinstein and Lewis, 1938). However, the mother exhibited no signs of this disease and birth was normal.

Very little can be said about the route of entry of the fungus into the brain. In view of the negative findings in the rest of the body there was no evidence of the infection being metastatic from some other organ. The sinuses and ears were not cultured for fungi at necropsy though they were grossly normal. It is possible that the ear or nasal sinuses were the portal of entry.

\section{Discussion}

Recorded examples of cerebral aspergillosis are few. Moniz and Loff (1931) reported the case of a 44-year-old woman in whom a complete right hemiplegia developed in 17 days. The cerebrospinal fluid was under normal pressure ; there were 60 cells per c. $\mathrm{mm}$., and the total protein was increased. At necropsy a large abscess was found in the left frontal lobe. Fungi, morphologically resembling aspergilli, were found in this abscess, but they were not cultured. The remainder of the 
post-mortem examination was negative. In view of the past history of corneal ulcer, the authors assumed that the fungus had entered the brain through the eye.

An aspergillus abscess and granuloma of the right frontal lobe was the subject of a report by Just (1931). The patient, a 52-year-old man, had had the clinical symptoms of a space-occupying lesion. Two operations were performed and at the second a culture of aspirated fluid revealed a pure growth of Aspergillus fumigatus. At necropsy the only significant finding was a lesion in the right frontal lobe. The orbital plate of the frontal bone on the right side was eroded in several places. Just considered that the infection had extended directly from the nose and frontal sinus through the frontal bone to the brain.

In 1935 Guillain, Bertrand, and Lereboullet described a case in which an abscess of the brain was presumably caused by aspergillus. The patient, a 33-year-old woman, presented the symptoms of a sphenoidal fissure syndrome on the right with pyramidal tract signs on the left. The disease had evolved over a period of one year. After various unsuccessful therapeutic measures she died, and at necropsy an abscess of the right frontal lobe was found. This abscess was surrounded by a dense, collagenous connective-tissue zone, and filamentous hyphae resembling aspergilli were found in the centre of the abscess. No culture was performed. There was localized chronic meningitis at the base of the skull, and the nerves in the right sphenoid fissure and the contents of the right orbit were involved in the pathological process. The authors stated that the fungus entered the cranial cavity by direct extension from the orbit.

Acute aspergillus meningitis in a 19-year-old man was reported by Linck (1939). His patient died five days after the onset of symptoms and at necropsy there was an acute exudative meningitis with several small granulomatous lesions along the Sylvian fissures. Mycelial threads which were identified as aspergillus were found in the centre of these granulomas. Cultures of the cerebrospinal fluid and meningeal exudate were not obtained.

The first case of generalized aspergillosis with involvement of the central nervous system was published by Cawley (1947). The patient, an 8-yearold child, had been ill since the age of 6 weeks with a chronic intermittent respiratory disease. He developed a multiloculated abscess of the right chest wall from which Aspergillus fumigatus was cultured. At the age of $7 \frac{1}{2}$ years signs of involvement of the cerebellum appeared, and a right cerebellar abscess was evacuated by operation. Following this, numerous abscesses formed all over the body and the patient finally succumbed. The necropsy revealed abscesses of varying size in the dura, brain, heart, lungs, mediastinum, lymph nodes, spleen, liver, right kidney, and right ankle. Aspergillus fumigatus was cultured from all the lesions at necropsy. It was concluded that the infection began in the lungs early in life and that the later clinical manifestations were the result of metastases.

A second instance of probable generalized aspergillosis, in a 22-year-old man, was recently reported by Grekin, Cawley, and Zheutlin (1950). The anatomical evidence of cerebral involvement is not convincing but a full pathological description of the case was not recorded.

McKee (1950) recorded the case of a basal meningitis in a 69-year-old man who had had symptoms of meningeal involvement for about two months. Several examinations of the cerebrospinal fluid revealed pleocytosis and an increase in the total protein content but cultures of the fluid were repeatedly negative. At necropsy a yellowish exudate was found at the base of the brain, and also in the sphenoid sinus. Upon microscopic examination there was chronic inflammation with large numbers of branching hyphae in both the brain and the wall of the sphenoid sinus. Cultures obtained after death, however, yielded only haemolytic Staphylococcus albus. McKee believed that the infection had spread directly from the sphenoid sinus to the base of the brain.

Cases of osteomyelitis of the base of the skull with a secondary localized granulomatous infection in the adjacent meninges have been reported by Nicod (1946) and Schnyder (1948). The last author quotes similar cases recorded by Oppe, and Wätjen. Fungi in the lesions of all these cases were identified morphologically as aspergilli, but they were not confirmed by culture.

Recently Wybel (1952) reported a pathologically verified case of aspergillus infection involving the meninges of the cervical spinal cord. His case is of particular interest in that the affection followed pneumococcal meningitis treated with intrathecal penicillin three years before.

Pathological Features. - In reviewing the cases that have been recorded in the literature, as well as the two cases of aspergillosis mentioned here, it becomes obvious that the pathological reaction in the nervous system takes the form of a chronic suppurative or granulomatous inflammation. The case reported by Linck (1939) is an exception, for in his case the organisms produced acute exudative meningitis with a predominantly polymorphonuclear reaction in the 
cerebrospinal fluid. It is also evident that the lesions may be localized either in the meninges or in the brain, the site depending upon the route of entry of the fungus. Even in the case where haematogenous dispersal of the fungi was proved (Cawley, 1947) the lesion took the form of localized abscesses in the cerebellum and in the meninges.

The cases quoted by Moniz and Loff (1931), Just (1931), Guillain and others (1935), Schnyder (1948), Nicod (1946), Wybel (1952), and our first case are examples of a localized productive inflammatory lesion. Our case is interesting in that the granulomatous meningeal mass was strictly localized to the posterior fossa and upper cervical spinal canal and produced the clinical picture of a slowly growing mass or an arachnoiditis involving the meninges and cervical roots. Similar clinical and pathological examples have been mentioned by Schnyder who cites cases of Wätjen and of Oppe. In their cases the primary lesion was a mycotic osteomyelitis of the base of the skull, with extension to the adjacent meninges and the formation of a localized meningeal granulomata ; Schnyder's case was mistaken clinically for tic douloreux because involvement of the Gasserian ganglion by the inflammatory mass had led to severe pain in the trigeminal distribution.

Our second case differs from others in that the brain was diffusely involved and pathologically the disease took the form of a granulomatous encephalitis with only a moderate reactive meningitis. The fungi had produced lesions over wide areas of the cerebral cortex and basal ganglia. These lesions had evolved slowly and permitted a dense fibrous giiosis with calcification to occur. Despite extensive involvement of the cerebral hemispheres there were no granulomatous lesions in the brain-stem and cerebellum. In a sense, therefore, the lesions, though diffuse, were localized in the cerebral hemispheres.

Mention should be made of the extensive calcification in the brain of the infant reported here. This led to the erroneous clinical diagnosis of toxoplasmosis, a condition in which cerebral calcification is quite common (Dyke, Wolf, Cowen, Paige, and Caffey, 1942). Calcifications of the brain may be encountered in a variety of other conditions (Courville and Adelstein, 1930). Calcifications in tuberculomata have been reported (Evans and Courville, 1938), and calcification in torulosis of the newborn has been reported by Neuhauser and Tucker (1948). Intracranial calcification following coccidioidal meningitis (Reeves and Baisinger, 1945) has also been reported. So far as we can determine calcification in aspergillus meningitis or encephalitis has not been described previously.
The possibility of this case being one of toxoplasmosis with secondary aspergillus infection was given serious consideration, but thought to be unlikely. A careful search of the lesions failed to demonstrate toxoplasma, but, more important, the character of the calcified and granulomatous lesions did not resemble those of toxoplasmosis.

Pathogenesis.-Deep fungus infections in general are known to produce either a chronic suppurative or granulomatous inflammatory response in tissue (Baker, 1947). The mechanism whereby fungi evoke this reaction is not fully understood, but it has been postulated that they act either as large foreign bodies or produce lesions by liberating toxic products. Henrici (1939) has demonstrated an endotoxin in Aspergillus fumigatus. Subcutaneous injection of this toxin into laboratory animals results in a severe local tissue necrosis and oedema. On intravenous administration degenerative and necrotic changes are seen in the liver and kidneys. The pathological changes in human aspergillosis, however, do not resemble those produced by the endotoxin in animals, but present, instead, the appearance of a foreign body reaction. Nevertheless, the variable reactions in individual cases raises the possibility of other mechanisms, as yet unknown, by which the observed tissue response is produced. The variability of pathological changes is illustrated in such different reactions as the acute exudative meningitis reported by Linck (1939) and the chronic fibrotic or calcific granulomatous lesions seen in the two cases reported here.

An interesting feature observed by Nicod (1946), Schnyder (1948), and McKee (1950) was the implication of the carotid artery by a granulomatous lesion. A similar type of involvement by Mucormyces was observed in the cases reported by Gregory, Golden, and Haymaker (1943) and LeCompte and Meissner (1947).

There has been much speculation and little actual data as to the route of entry of the fungi into the brain. Of the cases that have been reported in the literature only the one described by Cawley (1947) convincingly demonstrates a metastatic spread of the fungus to the brain. In his case fungi were demonstrated after death in the heart and lungs. Extension from the eye (Moniz and Loff, 1931), the orbit (Guillain and others, 1935), paranasal sinuses (Just, 1931 ; McKee, 1950 ; Nicod, 1946), lungs (Linck, 1939) have all been postulated but the pathological data often fail to establish the pathway. The route of entry of the fungus in the two cases reported here could not be definitely specified.

Wybel (1952) discusses the possible relationship, in 
his case, between intrathecal penicillin therapy and the development of cervical meningeal aspergillosis. $\mathrm{He}$ concludes that the growth of the fungus was enhanced by penicillin, and cites some evidence from the literature to support this view. In any event, lumbar puncture provided a probable route of entry for the fungus.

Clinical Features.-From the available clinical data it may be stated that aspergillosis of the central nervous system may result in several different syndromes. In seven of the 12 recorded cases there have been symptoms and signs of a solitary, localized, space-occupying intracranial lesion. In five of these the signs pointed to a disorder of the frontal pole of the cerebrum and in two to a posterior fossa lesion. Evidence of disease of retro-orbital structures, i.e. exophthalmos, ocular muscle palsies, amblyopia, and trigeminal nerve dysfunction, were observed in three of the five cases with involvement of the frontal lobes. In one case (Moniz and Loff, 1931) symptoms evolved over 17 days, but the others were chronic. Culture of the spinal fluid was attempted in only one of these cases and no growth was obtained. Lumbar punctures were performed on only three of the above cases and in each of them there were pleocytosis and elevated protein content.

Of the other five cases, one had clinical signs of acute leptomeningitis which led to death in five days, and another of a more chronic meningitis of several months' duration. In the case of Grekin, Cawley, and Zheutlin (1950) there was generalized aspergillosis and the symptoms of meningitis developed terminally. In one instance the disorder presented as a spinal cord tumour. Our second case is unique in that the principal clinical symptoms indicated cerebral disorder with dementia, seizures, and spastic paralysis of the arms and legs, and the onset of the disease was either prenatal or during the first few months after birth. The cerebrospinal fluid was not cultured in most of the cases of meningitis. It is probable that the organisms could have been recovered if this had been done.

\section{Summary}

Two new cases of aspergillus infection of the central nervous system are reported. In the first of these the symptoms, like those of several other previously published cases, were consistent with a chronic, localized granuloma of the meninges of the cervical cord and brain-stem. The second presented as chronic encephalitis with progressive dementia, convulsions, spastic paralysis, and cerebral calcification. The clinical manifestations, mycology, pathogenesis, and pathology of these and the other reported cases are discussed.

We wish to thank Dr. George Foley of the Children's Medical Centre, Boston, for his help in identifying the organism in Case 2.

\section{REFERENCES}

Baker, R. D. (1947). Arch. Path., Chicago, 44, 459.

Cawley, E. P. (1947). Arch. intern. Med., 80, 423.

Courville, C. B. (1945). Pathology of the central nervous system. $A$ study based upon a survey of lesions found in a series of thirty thousand autopsies, 2nd ed. Mountain View, California. Pp. 220-224.

Courville, C. B., and Adelstein, L. J. (1930). Arch. Surg., Chicago,

Dyke, C. G., Wolf, A., Cowen, D., Paige, B. H., and Caffey, J. (1942). Amer. J. Roentgenol., 47, 830.

Evans, H. S., and Courville, C. B. (1938). Arch. Surg., Chicago, 36,637 .

Goldstine, M. T. (1933). Amer. J. Obstet. Gynec., 25, 756.

Gregory, J. E., Golden, A., and Haymaker, W. (1943). Bull. Johns Hopk. Hosp., 73, 405.

Grekin, R. H., Cawley, E. P. and Zheutlin, B. (1950). Arch. Path., Chicago, 49, 387.

Guillain, G., Bertrand, I., and Lereboullet, J. (1935). Rev. neurol., Paris, 64, 684.

Henrici, A. T. (1939). J. Immunol., 36, 319.

Just, E. (1931). Mitt. Grenzgeb. Med. Chir., 42, 540.

LeCompte, P. M., and Meissner, W. A. (1947). Amer. J. Path., 23, 673 .

Linck, K. (1939). Virchows Arch. path. Anat., 304, 408.

McKee, E. E. (1950). Amer. J. clin. Path., 20, 381.

Moniz, E., and Loff, R. (1931). Presse méd., 39, 273.

Moore, M., Russell, W. O., and Sachs, E. (1946). Amer. J. Path.,

22, 761.
Neuhauser, E. B. D., and Tucker, A. (1948). Amer. J. Roentgenol., $59,805$.

Nicod, J. L. (1946). Schweiz. Z. Path. Bakt., 9, 673.

Oppe, -. (1897). Zbl. allg. Path. path. Anat., 8, 301 . Quoted by Schnyder.

Schnyder.
Reeves, D. L., and Baisinger, C. F. (1945). J. Neurosurg., 2, 269.
Schnyder, H. K. (1948). Pract. oto-rhino-laryng., Basel, 10, 402.

Thom, C., and Raper, K. B. (1945). A manual of the Aspergilli, p. 28. Baltimore.

Wätjen, J. (1928). Virchows Arch. path. Anat., 268, 665. Quoted by Schnyder.

Weinstein, L., and Lewis, R. M. (1938). Yale J. Biol. Med., 11, 85.

Whalen, E. J. (1938). J. Amer. med. Ass., 111, 502.

Wolf, A., and Cowen, D. (1937). Bull. neurol. Inst., N. Y., 6, 306. Wolf, A., Cowen, D., and Paige, B. H. (1939). Amer. J. Path., 15, 657.

Wybel, R. E. (1952). Arch. Path., Chicago., 53, 167. 